\title{
Federated Filter Based on Dynamic Information Allocation with Unscented Kalman Filter
}

\author{
Huang Jue ${ }^{1, a}$, Yan Bin ${ }^{1, b}$ \\ ${ }^{1}$ Department of Weaponry Engineering, Naval University of Engineering, Wuhan, Hubei, China \\ anudt_huangjue@163.com, biceyan@yeah.com
}

Keywords: Underwater wireless sensor, Dynamic information allocation, Federated filter, Soft failure sensitivity

\begin{abstract}
Because of the complex environment of the underwater noise and the difficulty for detecting the failure of the underwater sensors, a distributed filtering algorithm for the nonlinear target is proposed by applied the unscented kalman filter into the federated filter frame. Furthermore, the information allocation parameter is dynamically designed to be the ratio of the trace of the covariance matrix of the sub filter and the trace of the global covariance matrix. The soft failure sensitivity of the sub system is improved. The simulation result shows that the UKF federated filter can track the nonlinear target effectively and the soft failure sensitivity of the sub system can be identified easily.
\end{abstract}

\section{Introduction}

Multi-sensor integrated navigation has been widely used in the underwater sensor network. It improves the tracking precision of the single sensor and has better failure tolerance. There are two basic structures for the Multi-sensor data fusion: the centralized fusion and the distributed fusion. The centralized fusion collects all of the data to the fusion center to be processed, so there are not any losses of data. The centralized fusion is optimal. But the calculation and the communication burden of the centralized fusion may be too heavy and the failure tolerance is poor. The distributed fusion has got more and more attention in the recent years. N. A. Carlson proposed the federated filter in the 1990s[1]. The federated filter consists of a global filter and a number of local filters and the local filters are independent from each other, they apply their own filter algorithms, process own measurement information and generate the local tracking path. The global filter can only fuse the generated path of the local filters[2]. The traditional federated filter uses KF as its local filters, that leads the traditional federated filter can only track the linear moving targets. But often the measurement function is nonlinear; the KF can't use the nonlinear information, so the UKF is used as the local filter in the proposed algorithm. Furthermore, in order to identify the failure sensors, the dynamic information allocation is designed to be the ratio of the trace of the covariance matrix of the sub filter and the trace of the global covariance matrix. Simulation results indicate that the proposed algorithm can track the nonlinear system well and the accuracy is better than the UKF algorithm, and the soft failure sensitivity of the sub system can be enlarged, thus, the failure sensor can be identified easily.

\section{Federated UKF Based on Dynamic Information Allocation}

\section{Federated UKF.}

The federated kalman filter processes the measurement information from multi-sensors decentralized. Each local filter works parallelly and preprocesses its own measurements using kalman filter, and then sends the processed information to the global filter to be fused. The working process of federated kalman filter mainly consists of four procedures, those are the information allocation, time updated, measurement updated and information fusion. The local filter designed by N. A. Calson is the standard kalman filter. There are many restrictions in practice. In view of the nonlinear underwater target tracking problem, the UKF is used to replace the kalman filter for the local filter. Consider the dynamic system function: 


$$
x_{k+1}=f\left(x_{k}\right)+\Gamma_{k} w_{k}
$$

where, $x_{k} \in R^{n}$ is the n-dimensional state of the motion target at time step $k, \Gamma_{k} \in R^{n \times r}$ is the processing noise distribution matrix, $w_{k} \in R^{r}$ is the noise sequence with the covariance $Q_{k}$. Suppose that the noise at each time is independent with each other.

Suppose that there are $N$ sensors that observe the target, the measurement functions are as follows:

$$
z_{k+1}^{i}=h_{k+1}^{i}\left(x_{k+1}\right)+v_{k+1}^{i}, \quad i=1,2, \cdots N
$$

where $z_{k+1}^{i} \in R^{m}$ is the measurement value of sensor $i$ at time step $k+1$ and $v_{k+1}^{i} \in R^{m}$ is the corresponding measurement noise with its covariance being $R_{k+1}$ and mean being zero. All of the noises are supposed to be independence.

Suppose the initial state value of the global filter is $x_{0}^{g}$, the initial covariance is $P_{0}^{g}$, then according to the information allocation principle of the federated filter, we have:

$$
\begin{aligned}
& Q_{k}^{i}=\beta_{i}^{-1} Q_{k} \\
& P_{k \mid k}^{i}=\beta_{i}^{-1} P_{k \mid k}^{g} \\
& \hat{x}_{k \mid k}^{i}=\hat{x}_{k \mid k}^{g}
\end{aligned}
$$

Where $\beta_{i}>0$ is the information allocation parameter and:

$$
\sum_{i=1}^{N} \beta_{i}+\beta_{m}=1
$$

Let the results of the information allocation be the current value for the local filters. Each filter uses UKF to update the state, the UKF algorithm is as follows:

1. Time-update

Calculate sigma points:

$$
\xi_{k \mid k}=\left[\hat{x}_{k \mid k} \hat{x}_{k \mid k}+\sqrt{(n+\lambda) P_{k \mid k}} \hat{x}_{k \mid k}-\sqrt{(n+\lambda) P_{k \mid k}}\right]
$$

Compute the propagated sigma points:

$$
\gamma_{k \mid k}=f\left(\xi_{k \mid k}\right)
$$

Compute the predicted state and covariance:

$$
\begin{aligned}
& \hat{x}_{k+1 \mid k}=\sum_{i=0}^{2 n} w_{i}^{(m)} \gamma_{k \mid k, i} \\
& P_{k+1 \mid k}=\sum_{i=0}^{2 n} w_{i}^{(c)}\left(\gamma_{k \mid k, i}-\hat{x}_{k+1 \mid k}\right)\left(\gamma_{k \mid k, i}-\hat{x}_{k+1 \mid k}\right)^{T}+Q_{k}
\end{aligned}
$$

2. Measurement-update

Calculate sigma points:

$$
\xi_{k+1 \mid k}=\left[\hat{x}_{k+1 \mid k} \hat{x}_{k+1 \mid k}+\sqrt{(n+\lambda) P_{k+1 \mid k}} \hat{x}_{k+1 \mid k}-\sqrt{(n+\lambda) P_{k+1 \mid k}}\right]
$$

Compute the propagated sigma points:

$$
\gamma_{k+1 \mid k}=h\left(\xi_{k+1 \mid k}\right)
$$

Compute the predicted mean and the predicted covariance of the measurement and the cross-covariance of the state and the measurement:

$$
\begin{aligned}
& \hat{z}_{k+1 \mid k}=\sum_{i=0}^{2 n} w_{i}^{(m)} \gamma_{k+1 \mid k, i} \\
& P_{\tilde{z}_{k}}=\sum_{i=0}^{2 n} w_{i}^{(c)}\left(\gamma_{k+1 \mid k, i}-\hat{z}_{k+1 \mid k}\right)\left(\gamma_{k+1 \mid k, i}-\hat{z}_{k+1 \mid k}\right)^{T}+R_{k}
\end{aligned}
$$




$$
P_{\tilde{x}_{k} \tilde{z}_{k}}=\sum_{i=0}^{2 n} w_{i}^{(c)}\left(\xi_{k+1 \mid k, i}-\hat{x}_{k+1 \mid k}\right)\left(\gamma_{k+1 \mid k, i}-\hat{z}_{k+1 \mid k}\right)^{T}
$$

Compute the filter gain and the estimated state and its covariance:

$$
\begin{aligned}
& K_{k+1}=P_{\tilde{x}_{k} \tilde{z}_{k}} P_{\tilde{z}_{k}}^{-1} \\
& \hat{x}_{k+1 \mid k+1}=\hat{x}_{k+1 \mid k}+K_{k+1}\left(z_{k+1}-\hat{z}_{k+1 \mid k}\right) \\
& P_{k+1 \mid k+1}=P_{k+1 \mid k}-K_{k+1} P_{\tilde{z}_{k}}\left(K_{k+1}\right)^{T}
\end{aligned}
$$

Where

$$
\begin{aligned}
& \left\{\begin{array}{l}
w_{0}^{(m)}=\lambda /(n+\lambda) \\
w_{0}^{(c)}=\lambda /(n+\lambda)+\left(1-\alpha^{2}+\beta\right)
\end{array}\right. \\
& w_{i}^{(m)}=w_{i}^{(c)}=0.5 /(n+\lambda) \quad i=1,2, \ldots, n
\end{aligned}
$$

and $\lambda=\alpha^{2}(n+\kappa)-n$,often we choose $\alpha=0.01, \kappa=0, \beta=2$.

Then the local filters send the update results to the global filter to be fused. The fusion algorithm is:

$$
\begin{aligned}
& P_{k \mid k}^{g}=\left[\left(P_{k \mid k}^{1}\right)^{-1}+\left(P_{k \mid k}^{2}\right)^{-1}+\cdots+\left(P_{k \mid k}^{N}\right)^{-1}+\left(P_{k \mid k-1}^{m}\right)^{-1}\right]^{-1} \\
& \hat{X}_{k \mid k}^{g}=P_{k \mid k}^{g}\left[\left(P_{k \mid k}^{1}\right)^{-1} \hat{X}_{k \mid k}^{1}+\left(P_{k \mid k}^{2}\right)^{-1} \hat{x}_{k \mid k}^{2}+\cdots+\left(P_{k \mid k}^{N}\right)^{-1} \hat{X}_{k \mid k}^{N}+\left(P_{k \mid k-1}^{m}\right)^{-1} \hat{\chi}_{k \mid k-1}^{m}\right]
\end{aligned}
$$

\section{Dynamic Information Allocation.}

When the dimension and the transition matrix of the global filter and the local filters are the same, there will be few effect to the global optimal of the federated filter no matter what the value of $\beta_{i}$ is. But different $\beta_{i}$ will affect the accuracy and failure tolerance of the local filters. In practical application, the information allocation would satisfy that the higher accuracy the local filter gets, the larger parameter will be allocated. According to (21), we can get:

$$
\left(P_{k \mid k}^{g}\right)^{-1}=\left(P_{k \mid k}^{1}\right)^{-1}+\left(P_{k \mid k}^{2}\right)^{-1}+\cdots+\left(P_{k \mid k}^{N}\right)^{-1}+\left(P_{k \mid k-1}^{m}\right)^{-1}
$$

So, we have:

$$
\begin{aligned}
\operatorname{trace}\left(P_{k \mid k}^{g}\right)^{-1} & =\operatorname{trace}\left[\left(P_{k \mid k}^{1}\right)^{-1}+\left(P_{k \mid k}^{2}\right)^{-1}+\cdots+\left(P_{k \mid k}^{N}\right)^{-1}+\left(P_{k \mid k-1}^{m}\right)^{-1}\right] \\
& =\operatorname{trace}\left(P_{k \mid k}^{1}\right)^{-1}+\operatorname{trace}\left(P_{k \mid k}^{2}\right)^{-1}+\cdots+\operatorname{trace}\left(P_{k \mid k}^{N}\right)^{-1}+\operatorname{trace}\left(P_{k \mid k-1}^{m}\right)^{-1}
\end{aligned}
$$

We can choose:

$$
\begin{aligned}
& \beta_{i k}=\frac{\operatorname{trace}\left(P_{k \mid k}^{i}\right)^{-1}}{\operatorname{trace}\left(P_{k \mid k}^{g}\right)^{-1}} \\
& \beta_{m k}=\frac{\operatorname{trace}\left(P_{k \mid k-1}^{m}\right)^{-1}}{\operatorname{trace}\left(P_{k \mid k}^{g}\right)^{-1}}
\end{aligned}
$$

Noticed that:

$$
\begin{aligned}
\sum_{i=1}^{N} \beta_{i k}+\beta_{m k} & =\sum_{i=1}^{N} \frac{\operatorname{trace}\left(P_{k \mid k}^{i}\right)^{-1}}{\operatorname{trace}\left(P_{k \mid k}^{g}\right)^{-1}}+\frac{\operatorname{trace}\left(P_{k \mid k-1}^{m}\right)^{-1}}{\operatorname{trace}\left(P_{k \mid k}^{g}\right)^{-1}} \\
& =1
\end{aligned}
$$

So, this allocation method satisfies the principle of the information allocation.

\section{Simulation and Analysis}

Suppose the target moves with a constant angular velocity $\omega$, the state vector $X=[x, \dot{x}, y, \dot{y}]^{T}$, we can get the system function is:

$$
\dot{X}(t)=A(\omega) X(t)+B(\omega) w(t)
$$


Where

$$
A(\omega)=\left[\begin{array}{cccc}
0 & 1 & 0 & 0 \\
0 & 0 & 0 & -\omega \\
0 & 0 & 0 & 1 \\
0 & \omega & 0 & 0
\end{array}\right], B(\omega)=\left[\begin{array}{ll}
0 & 0 \\
1 & 0 \\
0 & 0 \\
0 & 1
\end{array}\right]
$$

and $w(t)$ is represented the process noise sequence.

We can get its discrete form as:

$$
X_{k+1}=\left[\begin{array}{cccc}
1 & \sin \frac{\omega T}{\omega} & 0 & -\frac{1-\cos \omega T}{\omega} \\
0 & \cos \omega T & 0 & -\sin \omega T \\
0 & \frac{1-\cos \omega T}{\omega} & 1 & \frac{\sin \omega T}{\omega} \\
0 & \sin \omega T & 0 & \cos \omega T
\end{array}\right] X_{k}+\left[\begin{array}{cc}
T^{2} / 2 & 0 \\
T & 0 \\
0 & T^{2} / 2 \\
0 & T
\end{array}\right] w_{k}
$$

and the covariance of the noise is $S_{k}$.

The measurement functions of the sensors are:

$$
z^{i}=h^{i}(x)+v^{i}
$$

where $h^{i}(x)=\left[h_{r}^{i}, h_{\theta}^{i}\right]^{T}$, and $v^{i}$ is the measurement noise, and

$$
\begin{aligned}
h_{r}^{i} & =\sqrt{\left(x_{m}-x_{i}\right)^{2}+\left(y_{m}-y_{i}\right)^{2}} \\
h_{\theta}^{i} & =\arctan \frac{y_{m}-y_{i}}{x_{m}-x_{i}}
\end{aligned}
$$

where $\left(x_{m}, y_{m}\right)$ is the position of the target, and $\left(x_{i}, y_{i}\right)$ is the position of the $i$ th sensor.

The values of the variables are:

\begin{tabular}{cccc}
\hline$\omega$ & $\mathrm{T}$ & $S_{k}$ & $\left(x_{m}^{i}, y_{m}^{i}\right)$ \\
\hline 0.03 & 0.1 & 0.1 & $(10,0),(0,10),(0,0),(10,10)$ \\
\hline
\end{tabular}

Suppose that the first sensor is a failure one in the system. The track of the failure sensor by two federated algorithm and the fusion track by the proposed algorithm after one time experiment running is as follow:

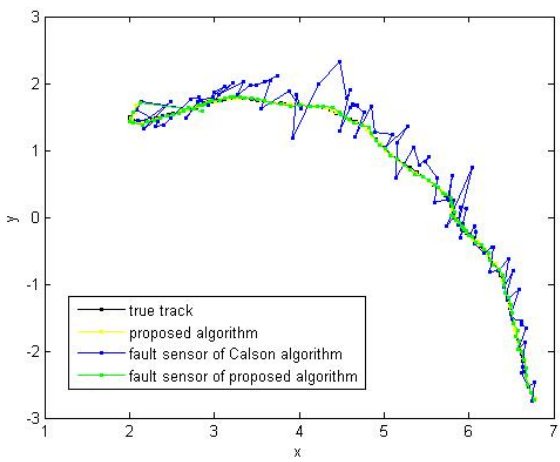

Fig 1 the track of the failure sensor and the fusion track

From the figure, we can see that the proposed algorithm can track the nonlinear system well, and the accuracy is higher than the single unscented kalman filter. The error of the failure sensor using the dynamic information allocation is larger than that using the fixed information allocation. Then the failure sensor can be located easily.

And after 50 times Monte-Carlo experiment, the root mean square error of the variables are as follows: 


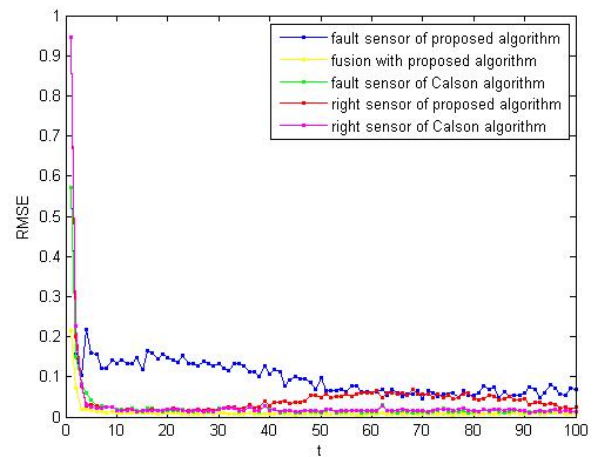

Fig 2 RMSE of x-position

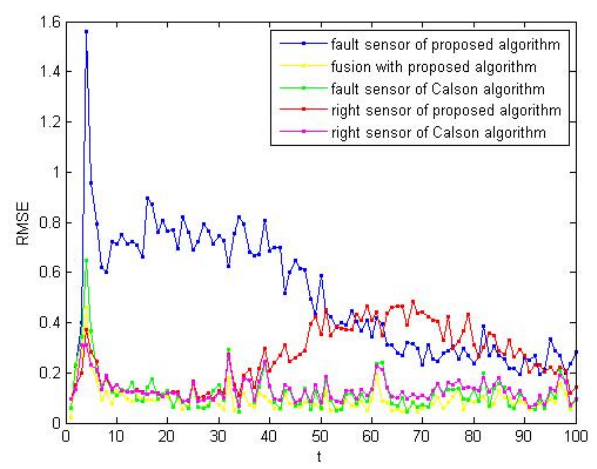

Fig 4 RMSE of x-velocity

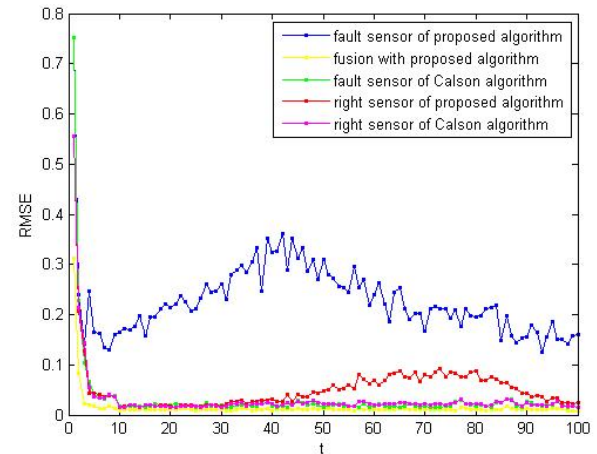

Fig 3 RMSE of y-position

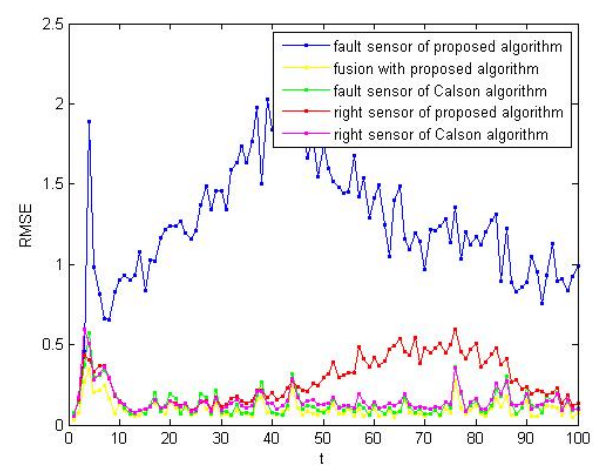

Fig 5 RMSE of y-velocity

We can see that when using fixed information allocation, because of the correction of the fusion results, the failure sensor almost has the same performance as the right sensors. So, the failure sensor can't be identified from the right sensors. The proposed dynamic information allocation algorithm reduces the feedback correction to the failure sensor by dynamicly adjusting the allocation parameters, so that the error of the failure sensor is obvious larger than the right sensors, and the failure sensor can be indentified easily.

\section{Summary}

In this paper, the nonlinear federated filter based on dynamic information allocation has been proposed. The unscented kalman filter is used as the local filter. And the information allocation parameter is determined by the trace of the local filter's covariance and the global filter's covariance. Simulation results show that the proposed algorithm can accurately track the nonlinear system; moreover the proposed algorithm increases the fault sensitivity of the local filters and can identify the failure sensor easily from the right sensors.

\section{References}

[1]. M. Kazerooni,A. Khayatian. Data Fusion for Autonomous Vehicle Navigation Based on Federated Filtering[C]. 2011 Australian Control Conference. 2011:368-373.

[2]. Jing Ma,Tong Wang,Zengping Wang,Thorp J S. Adaptive Damping Control of Inter-Area Oscillations Based on Federated Kalman Filter Using Wide Area Signals [J]. IEEE Transaction on Power Systems. 2013,28 (2):1627-1635.

[3]. Li Qiao,Jianye Liu,Guanglou Zheng,Zhi Xiong. Augmentation of XNAV System to an Ultraviolet Sensor-Based Satellite Navigation System [J]. IEEE Journal of Selected Topics in Signal Processing. 2009,3 (5):777-785. 
[4]. Wu XiaoJuan,Wang Xinlong. A SINS/CNS deep integrated navigation method based on mathematical horizon reference [J]. Aircraft Engineering and Aerospae Technology. 2011,83 (1):26-34.

[5]. Liu Jin,Ma Jie,Tian Jinwen. Pulsar/CNS integrated navigation based on federated UKF [J]. Journal of Systems Engineering and Electronics. 2010,21 (4):675-681.

[6]. Edemayer A,Miranda M,Nebehaj V. Cooperative federated filtering approach for enhanced position estimation and sensor fault tolerance in ad-hoc vehicle networks [J]. IET Intelligent Transport Systems. 2010,4 (1):82-92.

[7]. Maryam. Kazerooni,Faridoon Shabaninia,Mohanmad Vaziri,Suresh Vadhva. Federated Ensemble Kalman Filter in No Reset Mode Design[C]. 2013 IEEE IRI. August 14-16.2013:712-716.

[8]. Quan W,Fang J C,Xu F,Sheng W. Study for Hybrid simulation system of SINS/CNS integrated navigation [J]. IEEE A\&E System Magazine,2008,23 (2):17-24.

[9]. Xiaocheng Shi,Xingyan Sun,Mingyu Fu,Youzhen Chen and Wenbo Xie. Federated Filter for Multi-Sensor Data Fusion of Dynamic Positioning Ship[C]. Proceeding of IEEE International Conference on Automation and Logistics. 2012:13-18. 